\title{
Article \\ Cutoff Values of Hemodynamic Parameters in Pediatric Refractory Septic Shock
}

\author{
En-Pei Lee ${ }^{1,2} \mathbb{D}$, Jainn-Jim Lin ${ }^{1,2}$, Shao-Hsuan Hsia ${ }^{1,2}$, Oi-Wa Chan ${ }^{1,2}$, Sheng-Ling Jan ${ }^{3,4,5, *(\mathbb{D})}$ \\ and Han-Ping $\mathrm{Wu}^{6,7,8, *}$
}

1 Division of Pediatric Critical Care Medicine, Department of Pediatrics, Chang Gung Memorial Hospital at Linko, Kweishan, Taoyuan 333, Taiwan; pilichrislnp@gmail.com (E.-P.L.); lin0227@cgmh.org.tw (J.-J.L.); tw1picu002@gmail.com (S.-H.H.); oiwamail@gmail.com (O.-W.C.)

2 College of Medicine, Chang Gung University, Taoyuan 333, Taiwan

3 Department of Pediatrics, Children's Medical Center, Taichung Veterans General Hospital, Taichung City 407, Taiwan

4 School of Medicine, National Yang-Ming Chiao-Tung University, Taipei City 112, Taiwan

5 School of Medicine, Kaohsiung Medical University, Kaohsiung 80708, Taiwan

6 Department of Pediatric Emergency Medicine, Children's Hospital, China Medical University, Taichung 406040, Taiwan

7 Department of Medical Research, Children's Hospital, China Medical University, Taichung 406040, Taiwan

8 Department of Medicine, School of Medicine, China Medical University, Taichung 406040, Taiwan

* Correspondence: sljan@vghtc.gov.tw (S.-L.J.); arthur1226@gmail.com (H.-P.W.); Tel.: +886-4-23592525 (S.-L.J.); +886-4-22052121 (H.-P.W.)

check for updates

Citation: Lee, E.-P.; Lin, J.-J.; Hsia, S.-H.; Chan, O.-W.; Jan, S.-L.; Wu,

H.-P. Cutoff Values of Hemodynamic

Parameters in Pediatric Refractory Septic Shock. Children 2022, 9, 303. https://doi.org/10.3390/ children 9030303

Received: 6 January 2022

Accepted: 18 February 2022

Published: 22 February 2022

Publisher's Note: MDPI stays neutral with regard to jurisdictional claims in published maps and institutional affiliations.

Copyright: (C) 2022 by the authors. Licensee MDPI, Basel, Switzerland. This article is an open access article distributed under the terms and conditions of the Creative Commons Attribution (CC BY) license (https:// creativecommons.org/licenses/by/ $4.0 /)$.

\begin{abstract}
Background: Refractory septic shock can cause severe morbidities and mortalities in children. Resuscitation based on hemodynamics is important in children with critical illness. Thus, this study aimed to identify the hemodynamics of refractory septic shock associated with poor prognosis at an early stage to allow for timely interventions. Methods: We evaluated children with refractory septic shock admitted to a pediatric intensive care unit (PICU) and monitored their hemodynamics using a pulse index continuous cardiac output (PiCCO) system. The serial cardiac index (CI), systemic vascular resistance index (SVRI), and vasoactive-inotropic score (VIS) were recorded during the first $72 \mathrm{~h}$ after PICU admission. Results: Thirty-three children with refractory septic shock were enrolled. The SVRI and VIS were both associated with fatality from septic shock. The non-survivors had lower serial SVRI and higher VIS (both $p<0.05$ ). Based on the area under the ROC curve, the SVRI was the predictor during the early resuscitative stage (first $36 \mathrm{~h}$ ) in pediatric refractory septic shock. Conclusions: Both SVRI and VIS are predictors of mortality in children with refractory septic shock, and the SVRI is the powerful predictor of mortality in the early resuscitative stage. A low serial SVRI may allow for the early awareness of disease severity and strategies for adjusting vasoactive-inotropic agents to increase the SVRI.
\end{abstract}

Keywords: cutoff values; hemodynamic parameters; pediatric septic shock; outcomes; intensive care unit

\section{Introduction}

Circulatory shock refers to inappropriate perfusion that results in damage to tissues and causes mortality in children, which accounts for one-third of cases in intensive care units. According to the International Consensus Conference on Pediatric Sepsis, sepsis is defined as the systemic inflammatory response to a suspected or proven infection [1,2], and it can be graded on the basis of the severity and response to therapy, such as severe sepsis, septic shock, and refractory septic shock (RSS) [2]. The severity of sepsis from mild to severe was described previously, and the respective definitions are described as follows: severe sepsis (cardiovascular dysfunction, acute respiratory distress syndrome, or dysfunction in $\geq 2$ other organ systems), septic shock (sepsis with cardiovascular dysfunction which need 
vasoactive medication despite isotonic fluid resuscitation), and RSS (circulatory failure caused by septic cardiomyopathy despite fluid and vasoactive agents treatment) [2,3].

In children, sepsis remains the main cause of morbidity and mortality worldwide, and RSS is the most severe form of sepsis for which the rate of fatality ranges from $40 \%$ to 80\% [4,5]. At present, the Surviving Sepsis Campaign 2020 published the clinical standard for hemodynamic maintenance in children with sepsis [6]. In pediatric RSS, the important hemodynamic parameters are the cardiac index $(\mathrm{CI})$ and systemic vascular resistance index (SVRI). The therapeutic goal of CI 3.3-6.0 L/ $\mathrm{min} / \mathrm{m}^{2}$ may help achieve better outcomes [6-8]. Some reports have indicated that abnormal SVRI is associated with poor outcomes $[9,10]$. Furthermore, the vasoactive-inotropic score (VIS) is an equitable way of determining whether an individual requires vasoactive-inotropic drugs for cardiovascular support, which is also associated with prognosis $[3,10]$. Therefore, detecting abnormal hemodynamics in case of RSS at an early stage is important to allow for timely interventions with new vasoactive-inotropic agents to maintain threshold hemodynamics and improve clinical outcomes [11,12].

Few studies have investigated the systemic hemodynamics and VIS associated with 28-day mortality in children with RSS, and to our knowledge, no study has compared the performance of major hemodynamics, such as CI, SVRI, and VIS, to predict mortality in children with sepsis.

The pulse index continuous cardiac output (PiCCO) system incorporates transpulmonary thermodilution (TPTD), and it has shown effectiveness in monitoring the hemodynamics in children with critical illness. In this study, we aimed to analyze correlations between 28-day mortality and the most important hemodynamic parameters (i.e., serial CI and SVRI) and serial VIS in children with RSS.

\section{Materials and Methods}

\subsection{Patient Population}

Chart reviews of pediatric patients aged $<18$ years who had shock in a PICU were conducted from 2003 to 2017. A total of 29 beds were available in our PICU, and we treated patients aged from 1 month to 18 years. The study criteria, based on consensus definitions, were applied uniformly in our PICU, resulting in an internal standardized evaluation of the study [2].

Chang Gung Memorial Hospital's Institutional Review Board and Ethics Committee approved this study.

\subsection{Study Design}

The inclusion criterion was as follows: children with RSS who were admitted to the PICU. Fluid-RSS was constant shock regardless of the $60 \mathrm{~mL} / \mathrm{kg}$ of fluid resuscitation according to the guidelines of the Surviving Sepsis Campaign 2012 [3,13,14]. Catecholamineresistant shock was constant shock after fluid resuscitation and receiving one vasoactive medication [2,15]. Persistent catecholamine-resistant shock was constant shock despite receiving two kinds of vasoactive medications. Children with persistent catecholamineresistant shock were enrolled, and the hemodynamics was monitored via a PiCCO system (Pulsion Medical Systems, Munich, Germany). We gathered data pertaining to our patients: age; sex; baseline cardiac characteristics, such as initial inotropic equivalent, heart rate (beats/min), and mean arterial pressure (MAP; $\mathrm{mmHg}$ ); PiCCO system parameters; length of stay; and mortality.

Patients who met the criteria of persistent catecholamine-resistant shock were included, and they underwent PiCCO monitoring. Initial parameters included $72 \mathrm{~h}$ serial CI, SVRI, and VIS data, which were detected within $4 \mathrm{~h}$ of enrollment after PiCCO setup. Hemodynamics and VIS were recorded hourly after the PiCCO system was implemented. Hemodynamic parameters were further analyzed between the survivors and non-survivors to investigate whether serial CI, SVRI, and VIS could be early indicators for mortality in 
pediatric RSS. The two major clinical outcomes were defined as the 28-day mortality rate in the PICU and the length of ICU stay.

\subsection{Therapeutic Strategy}

We followed the guidelines of early goal-directed therapy (NEJM 2001) for children with septic shock. Resuscitation aimed to preserve the central venous pressure (CVP) of 8-12 mm Hg, adequate age-specific blood pressure (BP), cardiac index (CI) of $3.5-5.5 \mathrm{~L} / \mathrm{min} / \mathrm{m}^{2}$, and central venous saturation $(\mathrm{ScvO} 2) \geq 70 \%$. Fluid resuscitation $(10-20 \mathrm{~mL} / \mathrm{kg})$ is administered when the CVP level decreases; dopamine is administered when the systolic BP is not optimal (less than 5 th percentile for age), and inotropic agents are administered when the $\mathrm{CI}$ level declines, $\mathrm{ScvO} 2$ falls below $70 \%$, or serum lactate increases $(\geq 2 \mathrm{mmol} / \mathrm{L})[6,16]$.

\subsection{PiCCO Parameter Measurement}

Hemodynamics were continuously analyzed and output to a computer using PiCCOVoLEF Data Acquisition software (version 6.0; Pulsion Medical Systems, Bayern, Germany).

\subsection{Definition}

The VIS was defined as dopamine dose $(\mathrm{mcg} / \mathrm{kg} / \mathrm{min})+$ dobutamine dose $(\mathrm{mcg} / \mathrm{kg} / \mathrm{min})$ $+100 \times$ epinephrine dose $(\mathrm{mcg} / \mathrm{kg} / \mathrm{min})+100 \times$ norepinephrine dose $(\mathrm{mcg} / \mathrm{kg} / \mathrm{min}) 10 \times$ milrinone dose $(\mathrm{mcg} / \mathrm{kg} / \mathrm{min})+10,000 \times$ vasopressin dose (units $/ \mathrm{kg} / \mathrm{min}$ ) [17].

\subsection{Statistical Analysis}

Multivariate logistic regression analysis, the chi-square test, Fisher's exact test, Student's $t$-test, and the Mann-Whitney $U$ test were all conducted. Descriptive analysis used means \pm standard deviations to show the value. The chi-square or Fisher's exact tests were conducted for comparison of dichotomous variables between the two groups. As a final step, the optimal cutoff values of hemodynamic parameters to predict mortality were determined using the receiver operating characteristic (ROC) curve. LR+ and LRwere calculated to obtain the best cutoff values. Likewise, DeLong's test was conducted to determine whether a significant difference in AUCs of the hemodynamic parameters exists. Significance was set at $p<0.05$. Statistics were conducted utilizing SPSS (version 22.0; SPSS Inc., Chicago, IL, U.S.A.).

\section{Results}

\subsection{Demographic Characteristics of Pediatric Septic Shock}

Over the course of the 15-year study period, 11,832 patients were admitted to our PICU, of whom 2699 (22.8\%) suffered from sepsis. Septic shock was observed in 520 $(19.2 \%)$ of the 2699 patients with sepsis. The PiCCO device was inserted for invasive hemodynamic monitoring in 39 children with persistent catecholamine-resistant shock. As six patients had missing data, we finally enrolled 33 patients for further analysis (Figure 1). Among the enrolled children, $72.8 \%$ were microbiologically confirmed as having an infection. The bloodstream was the most frequently observed site of infection, with Gram-negative bacteria as the predominant pathogen. The patient survival rate was $45 \%$, and the mortality rate was $55 \%$. Both survival and mortality groups commonly used dopamine and epinephrine as initial vasoactive-inotropic agents. After the PiCCO arrangement, the non-survivors exhibited a significantly higher VIS but a significantly lower MAP, compared with the survivors $(p<0.05)$. 


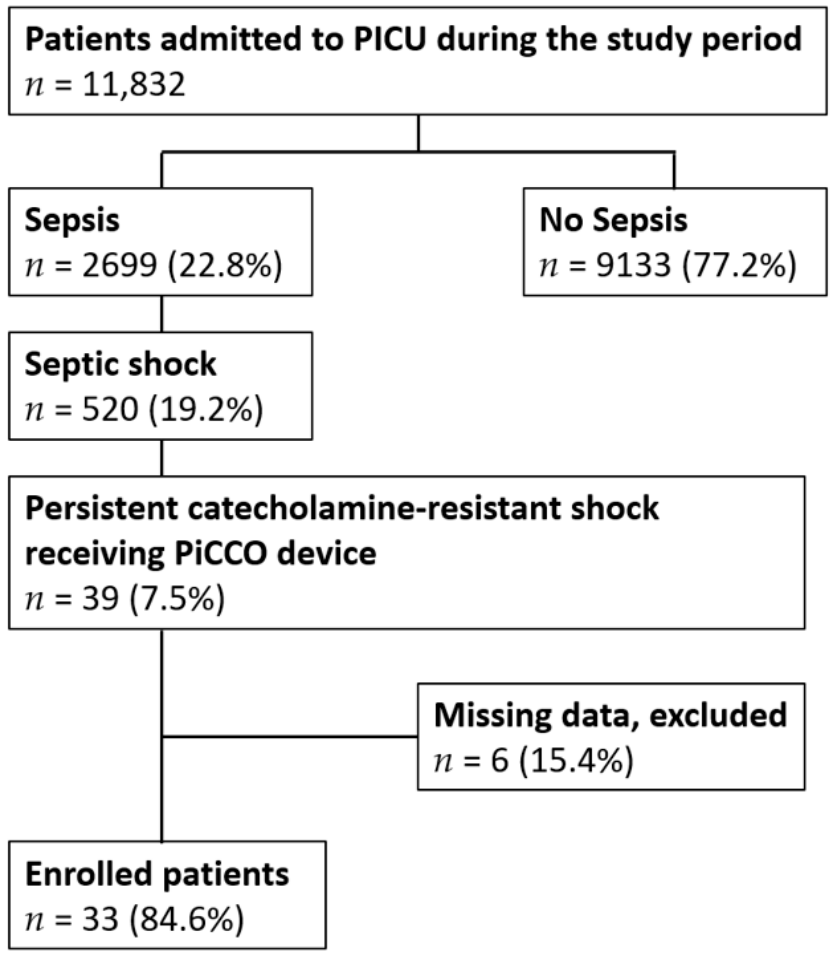

Figure 1. Algorithm of enrolled patients.

\subsection{PiCCO Parameters after Setting Up TPTD}

$\mathrm{CO}$ and cardiac contractility parameters measured by PiCCO showed no significant difference between survivors and non-survivors (Table 1). A higher SVV (a preload parameter) and lower SVRI (an afterload parameter) were observed in the non-survivors compared with the survivors (both $p<0.05$ ).

Table 1. Population characteristics and initial PiCCO parameters.

\begin{tabular}{lccc}
\hline \multicolumn{1}{c}{ Variables } & Survival $(\mathbf{n}=\mathbf{1 5})$ & 28-Day Mortality $(\mathbf{n}=\mathbf{1 8})$ & $p$ Value \\
\hline Age (years) & $12.1 \pm 4.6$ & $12.3 \pm 4.3$ & 0.786 \\
Gender (male), $\mathrm{n}(\%)$ & $6(40)$ & $10(55.6)$ & 0.373 \\
Weight $(\mathrm{kg})$ & $34.5 \pm 15.1$ & $36.6 \pm 15$ & 0.703 \\
Underlying, $\mathrm{n}(\%)$ & $8(53.3)$ & $13(72.2)$ & 0.446 \\
PRISM score & $18.3 \pm 4.9$ & $19.7 \pm 3.1$ & 0.329 \\
Site of infection, $\mathrm{n}(\%)$ & & & 0.18 \\
$\quad$ CNS & 0 & $5(27.8)$ & $7(38.8)$ \\
Bloodstream & $6(40)$ & $3(16.7)$ & 0.77 \\
Respiratory & $4(26.6)$ & 0 & 0.513 \\
Urologic & $1(6.7)$ & 0 & \\
Abdominal & $1(6.7)$ & $3(16.7)$ & \\
Others & $3(20)$ & $13(72.3)$ & \\
Culture positive, $\mathrm{n}(\%)$ & $11(73.3)$ & $2(11.1)$ & \\
Pathogen, $\mathrm{n}(\%)$ & & $8(44.4)$ & \\
$\quad$ Gram positive & $5(33.3)$ & $2(5.6)$ & \\
Gram negative & $5(33.3)$ & $5(27.7)$ & \\
Fungus & $1(6.8)$ & $0(0)$ & \\
$\quad$ Virus & $4(26.6)$ & & \\
$\quad$ Unknown & &
\end{tabular}


Table 1. Cont.

\begin{tabular}{|c|c|c|c|}
\hline Variables & Survival (n = 15) & 28-Day Mortality $(\mathrm{n}=18)$ & $p$ Value \\
\hline \multicolumn{4}{|c|}{ Used vasoactive-inotropic agents, $\mathrm{n}(\%)$} \\
\hline Dopamine & $14(93.3)$ & $18(100)$ & 0.9 \\
\hline Epinephrine & $12(80)$ & $17(94.4)$ & 0.46 \\
\hline Norepinephrine & $5(33.3)$ & $9(50)$ & 0.543 \\
\hline Dobutamine & $1(6.7)$ & $7(38.9)$ & 0.08 \\
\hline Milrinone & $10(66.7)$ & $10(55.6)$ & 0.76 \\
\hline Vasopressin & 0 & $1(5.5)$ & 0.9 \\
\hline \multicolumn{4}{|l|}{ Resuscitative IV fluid (mL/kg) } \\
\hline $\begin{array}{l}\text { IV fluid prior to the study } \\
\text { included }\end{array}$ & $59.7 \pm 5.3$ & $60.1 \pm 6.2$ & 0.81 \\
\hline $\begin{array}{l}\text { IV fluid included in the study } \\
\text { within } 72 \mathrm{~h}\end{array}$ & $17.2 \pm 4.6$ & $15.5 \pm 1.2$ & 0.164 \\
\hline \multicolumn{4}{|l|}{ Cardiac characteristics } \\
\hline VIS & $27.5 \pm 12.3$ & $54.7 \pm 56$ & 0.015 \\
\hline Heart rate (beats/min) & $120.3 \pm 29.9$ & $142.4 \pm 24.7$ & 0.057 \\
\hline Mean arterial pressure (mm Hg) & $83.5 \pm 16.2$ & $58.8 \pm 16.7$ & $<0.001$ \\
\hline \multicolumn{4}{|l|}{ Outcomes } \\
\hline ICU stay (days) & $22.1 \pm 15.7$ & $13.8 \pm 7.7$ & 0.057 \\
\hline Length of stay (days) & $37.3 \pm 28.1$ & $22.3 \pm 16$ & 0.093 \\
\hline \multicolumn{4}{|l|}{ Initial PiCCO data } \\
\hline \multicolumn{4}{|l|}{ Cardiac output } \\
\hline $\mathrm{CO}(\mathrm{L} / \mathrm{min})$ & $4.4 \pm 1.7$ & $4.7 \pm 1.7$ & 0.628 \\
\hline \multicolumn{4}{|l|}{ Cardiac contractility } \\
\hline $\mathrm{CI}\left(\mathrm{L} / \mathrm{min} / \mathrm{m}^{2}\right)$ & $3.9 \pm 1.1$ & $4.1 \pm 0.9$ & 0.602 \\
\hline GEF $(\%)$ & $31.3 \pm 10.1$ & $27.8 \pm 9.9$ & 0.502 \\
\hline CFI (I/min) & $9.2 \pm 2.8$ & $9.4 \pm 3.4$ & 1 \\
\hline \multicolumn{4}{|l|}{ Preload parameters } \\
\hline $\operatorname{GEDVI}\left(\mathrm{mL} / \mathrm{m}^{2}\right)$ & $451.8 \pm 154.2$ & $457.5 \pm 145.2$ & 0.823 \\
\hline $\operatorname{ITBVI}\left(\mathrm{mL} / \mathrm{m}^{2}\right)$ & $564.2 \pm 192.8$ & $571.5 \pm 181.5$ & 0.823 \\
\hline SVV $(\%)$ & $9.7 \pm 3.3$ & $16.1 \pm 6.7$ & 0.003 \\
\hline \multicolumn{4}{|l|}{ Afterload parameter } \\
\hline SVRI $\left(\mathrm{dyn} \times \mathrm{sec} / \mathrm{cm}^{5} / \mathrm{m}^{2}\right)$ & $1413.1 \pm 537.7$ & $933.9 \pm 376.9$ & 0.003 \\
\hline \multicolumn{4}{|l|}{ Lung parameters } \\
\hline EVLWI $\left(\mathrm{mL} / \mathrm{m}^{2}\right)$ & $10.7 \pm 4.7$ & $15.9 \pm 14.9$ & 0.551 \\
\hline PVPI & $2.9 \pm 0.9$ & $3.9 \pm 3.1$ & 0.502 \\
\hline
\end{tabular}

PRISM, pediatric risk of mortality; CNS, central nervous system; IV, intravascular. VIS, vasoactive-inotropic scores, ICU, intensive care unit; $\mathrm{CO}$, cardiac output; $\mathrm{CI}$, cardiac index; GEF, global ejection fraction; $\mathrm{CFI}$, cardiac function index; GEDVI, global end-diastolic volume index; ITBVI, intrathoracic blood volume index; SVV, stroke volume variation; SVRI, systemic vascular resistance index; EVLWI, extravascular lung water index; PVPI, pulmonary vascular permeability index.

\subsection{Factors Associated with 28-Day Mortality}

After the PiCCO device were implemented, the CI was measured every $6 \mathrm{~h}$ (Figure 2). Only 33 cases were identified in this study; thus, three important parameters (CI, SVRI, and VIS) were put in the multivariate logistic regression analysis. The results reported that the SVRI and VIS were independent predictors of 28-day mortality during the first $72 \mathrm{~h}$ after admission to the PICU (Table 2). Table 3 summarizes the AUC and cutoff values of $\mathrm{CI}, \mathrm{SVRI}$, and VIS for the survival and mortality groups every $6 \mathrm{~h}$. Within the first $36 \mathrm{~h}$ after patients were enrolled, the average AUC of the SVRI was the highest (0.83) among all predictors, and within $42-54 \mathrm{~h}$, the VIS showed the highest average AUC (0.77); however, within 60-66 h, the SVRI showed the highest average AUC (0.78). The serial SVRI measured every $6 \mathrm{~h}$ was lower in the non-survivors, which was significantly lower within $0-42 \mathrm{~h}$ and at the 60th hour (Figure 3). The serial VIS was higher in the mortality group, particularly within $0-48 \mathrm{~h}$, except at the 12th hour (Figure 4). Figures 5 and 6 depict the ROC analysis for CI, SVRI, and VIS calculated every $6 \mathrm{~h}$ when attempting to predict survival, and Table 4 provides the best CI cutoff values for each predictor. DeLong's test showed that the AUC 
of the SVRI was better than that of the CI $(p<0.05)$, but no significant difference was noted between VIS and CI. Furthermore, the two cutoff values of $100 \%$ specificity and $100 \%$ sensitivity for the SVRI in predicting clinical outcomes at $6 \mathrm{~h}$ interval are shown in Table 5.



Figure 2. Serial cardiac index (CI) and its variance (mean $\pm 1 \mathrm{SE}$ bar) after comparing survivors with non-survivors, measured at $6 \mathrm{~h}$ intervals.

Table 2. Multivariable analysis for the predictors of the 28-day mortality during the first $72 \mathrm{~h}$ after admission to the PICU.

\begin{tabular}{cccc}
\hline Parameter & $\boldsymbol{\beta}$ & Odds Ratio & $p$ Value \\
\hline $\mathrm{CI}\left(\mathrm{L} / \mathrm{min} / \mathrm{m}^{2}\right)$ & -0.18 & $0.836(0.334-2.092)$ & 0.701 \\
VIS & 0.057 & $1.058(1.001-1.119)$ & $0.048^{*}$ \\
SVRI & -0.003 & $0.997(0.995-0.999)$ & 0.028 *
\end{tabular}

VIS, vasoactive-inotropic score; CI, cardiac index; SVRI, systemic vascular resistance index. ${ }^{*}$ Statistical significance was set at $p<0.05$. 
Table 3. Comparison of CI, SVRI, VIS, and ROC at six-hour intervals between survivors and nonsurvivors.

\begin{tabular}{|c|c|c|c|c|c|c|c|c|c|}
\hline \multirow[b]{2}{*}{ Hour } & \multicolumn{3}{|c|}{$\mathrm{CI}\left(\mathrm{L} / \mathrm{min} / \mathrm{m}^{2}\right)$} & \multicolumn{3}{|c|}{ SVRI $\left(\right.$ dyn $\left.\times \mathrm{sec} / \mathrm{cm}^{5} / \mathrm{m}^{2}\right)$} & \multicolumn{3}{|c|}{ VIS } \\
\hline & Survival & $\begin{array}{c}\text { 28-Day } \\
\text { Mortality }\end{array}$ & AUC & Survival & $\begin{array}{c}\text { 28-Day } \\
\text { Mortality }\end{array}$ & AUC & Survival & $\begin{array}{c}\text { 28-Day } \\
\text { Mortality }\end{array}$ & AUC \\
\hline 0 & $3.9 \pm 1.2$ & $4.1 \pm 1.3$ & 0.51 & $1413 \pm 537$ & $933 \pm 376$ & 0.8 & $28 \pm 13$ & $58 \pm 55$ & 0.76 \\
\hline 6 & $3.8 \pm 1.2$ & $4 \pm 1.1$ & 0.46 & $1543 \pm 645$ & $1019 \pm 354$ & 0.76 & $36 \pm 22$ & $69 \pm 62$ & 0.73 \\
\hline 12 & $3.9 \pm 1$ & $4.4 \pm 1.2$ & 0.32 & $1594 \pm 552$ & $935 \pm 261$ & 0.89 & $38 \pm 26$ & $66 \pm 50$ & 0.71 \\
\hline 18 & $4.1 \pm 1.4$ & $4.9 \pm 1.3$ & 0.34 & $1731 \pm 583$ & $851 \pm 394$ & 0.88 & $36 \pm 26$ & $79 \pm 57$ & 0.73 \\
\hline 24 & $4.2 \pm 1.5$ & $3.9 \pm 1$ & 0.47 & $1697 \pm 483$ & $1040 \pm 386$ & 0.84 & $25 \pm 14$ & $60 \pm 35$ & 0.79 \\
\hline 30 & $4.1 \pm 1.1$ & $4.2 \pm 0.9$ & 0.44 & $1602 \pm 511$ & $957 \pm 322$ & 0.83 & $23 \pm 16$ & $52 \pm 32$ & 0.79 \\
\hline 36 & $4.4 \pm 1.3$ & $4.1 \pm 0.9$ & 0.54 & $1631 \pm 488$ & $1099 \pm 551$ & 0.79 & $22 \pm 16$ & $46 \pm 27$ & 0.76 \\
\hline 42 & $3.7 \pm 1.2$ & $3.6 \pm 0.8$ & 0.51 & $1886 \pm 627$ & $1290 \pm 658$ & 0.77 & $21 \pm 15$ & $49 \pm 28$ & 0.79 \\
\hline 48 & $3.8 \pm 1.3$ & $3.7 \pm 0.9$ & 0.52 & $1973 \pm 577$ & $1424 \pm 613$ & 0.76 & $21 \pm 15$ & $53 \pm 36$ & 0.78 \\
\hline 54 & $4 \pm 1.3$ & $4.4 \pm 1.3$ & 0.38 & $1768 \pm 554$ & $1345 \pm 615$ & 0.62 & $22 \pm 13$ & $39 \pm 23$ & 0.73 \\
\hline 60 & $3.8 \pm 1.5$ & $4.2 \pm 0.9$ & 0.35 & $1866 \pm 639$ & $1149 \pm 638$ & 0.8 & $22 \pm 12$ & $45 \pm 31$ & 0.73 \\
\hline 66 & $3.4 \pm 0.8$ & $3.8 \pm 1.2$ & 0.4 & $1951 \pm 564$ & $1405 \pm 705$ & 0.75 & $21 \pm 13$ & $39 \pm 29$ & 0.70 \\
\hline 72 & $3.7 \pm 1.1$ & $3.9 \pm 1.4$ & 0.52 & $1766 \pm 439$ & $1489 \pm 880$ & 0.62 & $21 \pm 13$ & $39 \pm 29$ & 0.70 \\
\hline
\end{tabular}

CI, cardiac index; SVRI, systemic vascular resistance index; VIS, vasoactive-inotropic score; AUC, area under the ROC curve; ROC, receiver operating characteristic.

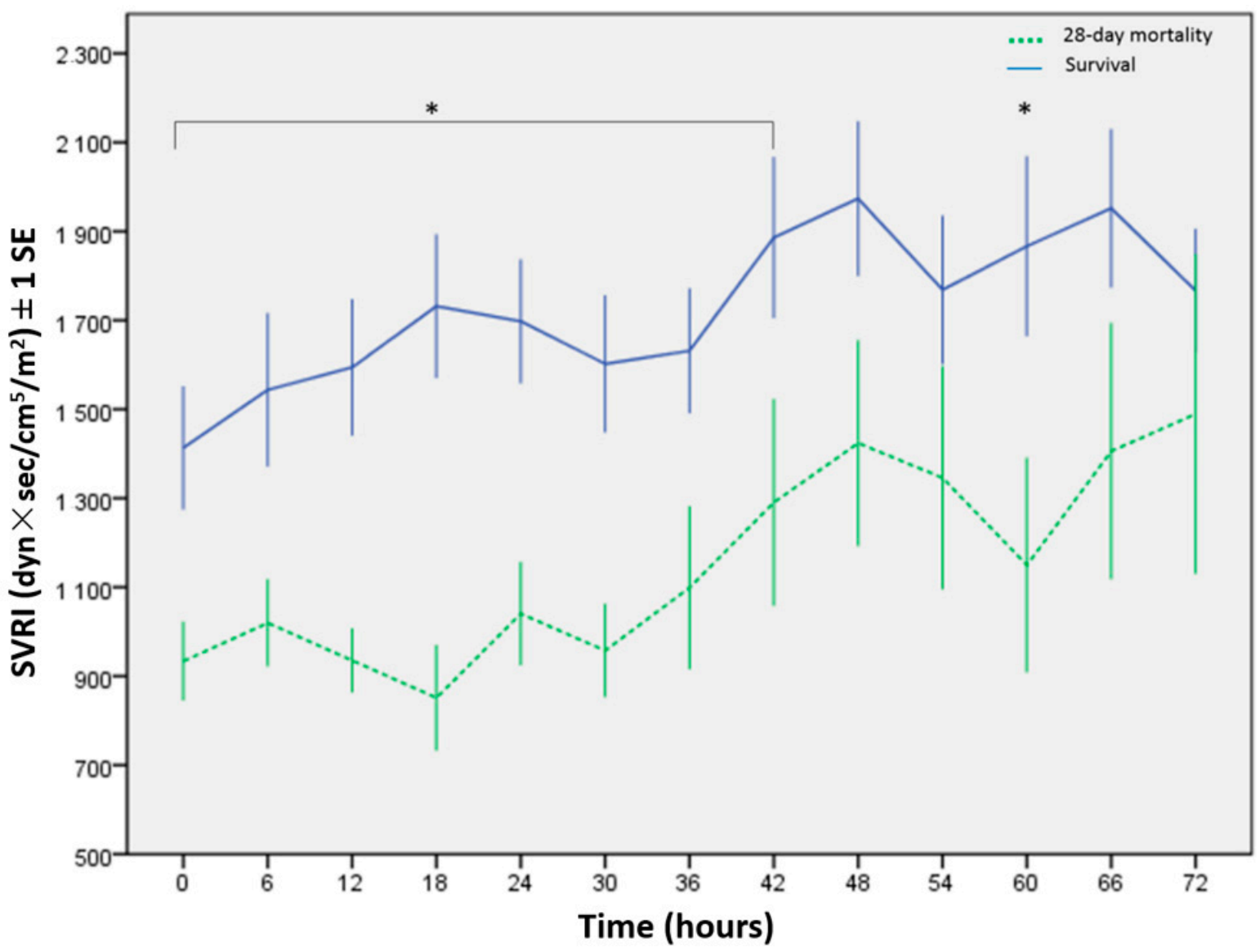

Figure 3. Serial systemic vascular resistance index (SVRI), measured at $6 \mathrm{~h}$ intervals. * Statistical significance was set at $p<0.05$. 


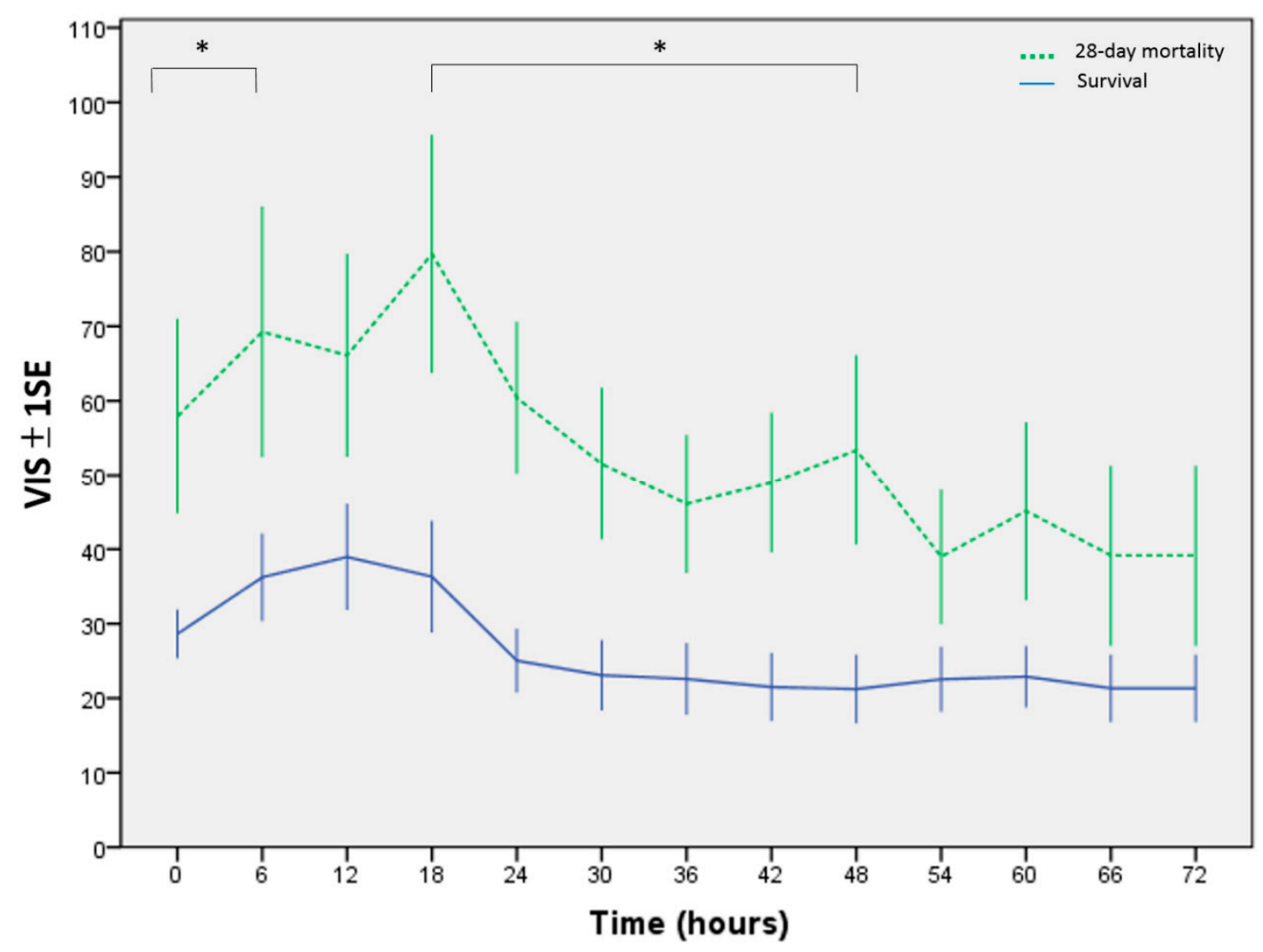

Figure 4. Serial vasoactive-inotropic score (VIS), measured at $6 \mathrm{~h}$ intervals. ${ }^{*}$ Statistical significance was set at $p<0.05$.
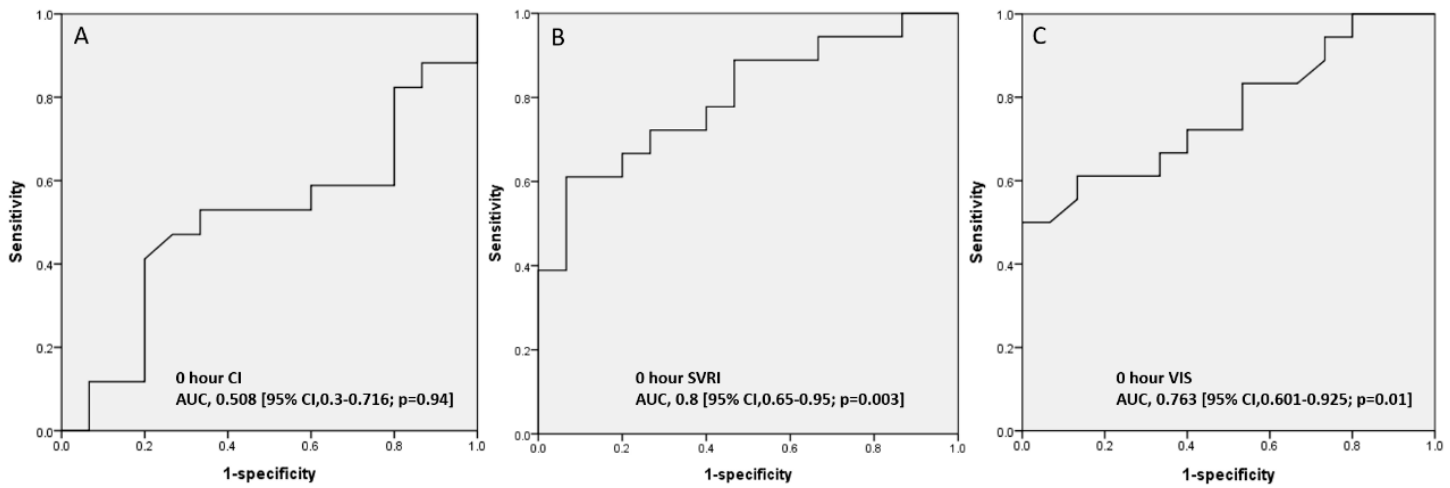

Figure 5. Predicting 28-day mortality at $0 \mathrm{~h}$ based on the AUCs of CI, SVRI, and VIS. (A) CI, (B) SVRI, and (C) VIS. 

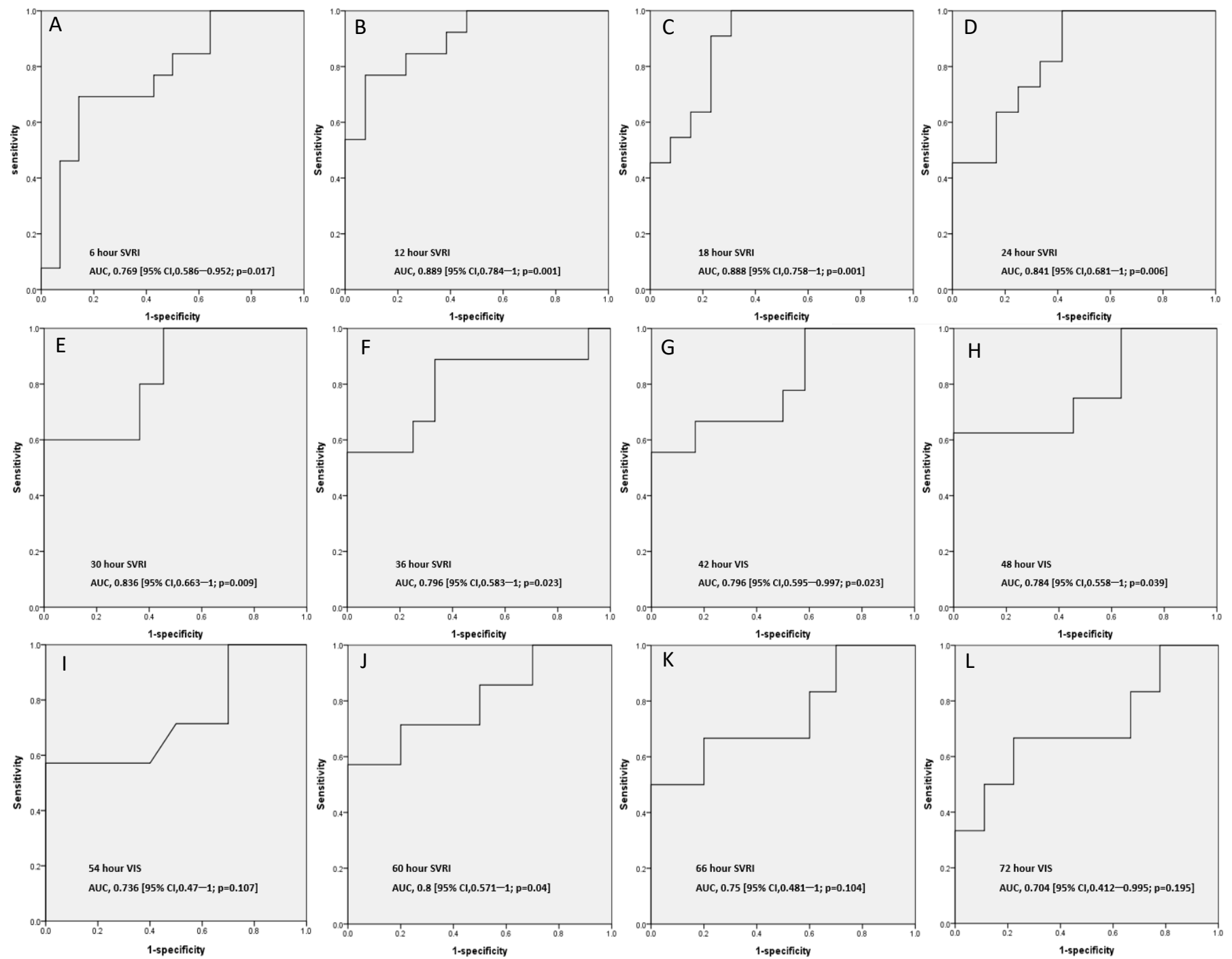

Figure 6. Predicting 28-day mortality at $6 \mathrm{~h}$ intervals using the best AUC. (A-L) 6-72 h.

Table 4. Best cutoff values of hemodynamic parameters at $6 \mathrm{~h}$ intervals within $72 \mathrm{~h}$ after the PiCCO setup.

\begin{tabular}{cccccccc}
\hline $\begin{array}{c}\text { Time } \\
\text { (Hours) }\end{array}$ & $\begin{array}{c}\text { Hemodynamic } \\
\text { Parameters }\end{array}$ & Cutoff Value & Sensitivity & Specificity & LR $^{+}$ & LR $^{-}$ & $\begin{array}{c}\text { Youden } \\
\text { Index }\end{array}$ \\
\hline 0 & SVRI & 896 & 0.61 & 0.93 & 9.2 & 0.4 & 0.5 \\
6 & SVRI & 1011 & 0.69 & 0.86 & 4.9 & 0.4 & 0.5 \\
12 & SVRI & 978 & 0.77 & 0.92 & 10 & 0.3 & 0.7 \\
18 & SVRI & 1294 & 0.91 & 0.77 & 3.9 & 0.1 & 0.7 \\
24 & SVRI & 1284 & 0.73 & 0.75 & 2.9 & 0.4 & 0.5 \\
30 & SVRI & 1014 & 0.6 & 0.9 & 6.6 & 0.4 & 0.5 \\
36 & SVRI & 1446 & 0.89 & 0.67 & 2.7 & 0.1 & 0.6 \\
42 & VIS & 49 & 0.56 & 1 & - & 0.4 & 0.6 \\
48 & VIS & 44 & 0.63 & 1 & - & 0.3 & 0.6 \\
54 & VIS & 42 & 0.58 & 1 & - & 0.4 & 0.6 \\
60 & SVRI & 1312 & 0.7 & 0.8 & 3.5 & 0.4 & 0.5 \\
66 & SVRI & 1454 & 0.67 & 0.8 & 3.3 & 0.4 & 0.6 \\
72 & VIS & 27 & 0.67 & 0.78 & 3 & 0.4 & 0.4 \\
\hline
\end{tabular}


Table 5. Two cutoff values for SVRI in predicting clinical outcomes with $100 \%$ specificity and $100 \%$ sensitivity at $6 \mathrm{~h}$ intervals.

\begin{tabular}{|c|c|c|c|c|c|c|}
\hline $\begin{array}{c}\text { Time } \\
\text { (Hours) }\end{array}$ & SVRI & Sensitivity & Specificity & $\mathbf{L R}^{+}$ & $\mathbf{L R}^{-}$ & $\begin{array}{c}\text { Youden } \\
\text { Index }\end{array}$ \\
\hline 0 & 533 & $20 \%$ & $100 \%$ & - & 0.8 & 0.2 \\
\hline 0 & 1531 & $100 \%$ & $10 \%$ & 1.1 & 0 & 0.1 \\
\hline 6 & 1011 & $70 \%$ & $100 \%$ & - & 0.3 & 0.7 \\
\hline 6 & 1789 & $100 \%$ & $40 \%$ & 1.7 & 0 & 0.4 \\
\hline 12 & 837 & $60 \%$ & $100 \%$ & - & 0.4 & 0.6 \\
\hline 12 & 1492 & $100 \%$ & $70 \%$ & 3.3 & 0 & 0.7 \\
\hline 18 & 914 & $60 \%$ & $100 \%$ & - & 0.4 & 0.6 \\
\hline 18 & 1534 & $100 \%$ & $70 \%$ & 3.3 & 0 & 0.7 \\
\hline 24 & 1156 & $60 \%$ & $100 \%$ & - & 0.4 & 0.6 \\
\hline 24 & 1590 & $100 \%$ & $70 \%$ & 3.3 & 0 & 0.7 \\
\hline 30 & 959 & $60 \%$ & $100 \%$ & - & 0.4 & 0.6 \\
\hline 30 & 1646 & $100 \%$ & $70 \%$ & 3.3 & 0 & 0.7 \\
\hline 36 & 1004 & $40 \%$ & $100 \%$ & - & 0.6 & 0.4 \\
\hline 36 & 2390 & $100 \%$ & $10 \%$ & 1.1 & 0 & 0.1 \\
\hline 42 & 1033 & $50 \%$ & $100 \%$ & - & 0.5 & 0.5 \\
\hline 42 & 2400 & $100 \%$ & $30 \%$ & 1.4 & 0 & 0.3 \\
\hline 48 & 1100 & $40 \%$ & $100 \%$ & - & 0.6 & 0.4 \\
\hline 48 & 2487 & $100 \%$ & $40 \%$ & 1.7 & 0 & 0.4 \\
\hline 54 & 997 & $30 \%$ & $100 \%$ & - & 0.7 & 0.3 \\
\hline 54 & 2000 & $100 \%$ & $20 \%$ & 1.3 & 0 & 0.2 \\
\hline 60 & 1063 & $60 \%$ & $100 \%$ & - & 0.4 & 0.6 \\
\hline 60 & 2327 & $100 \%$ & $40 \%$ & 1.7 & 0 & 0.4 \\
\hline 66 & 1106 & $50 \%$ & $100 \%$ & - & 0.5 & 0.5 \\
\hline 66 & 2494 & $100 \%$ & $40 \%$ & 1.7 & 0 & 0.4 \\
\hline 72 & 1151 & $50 \%$ & $100 \%$ & - & 0.5 & 0.5 \\
\hline 72 & 2795 & $100 \%$ & 0 & 1 & 0 & 0 \\
\hline
\end{tabular}

\section{Discussion}

Septic shock has a high in-hospital mortality rate, particularly for children who experience RSS. Hemodynamic monitoring is essential for the evaluation and therapeutic management of patients with critical illness, especially for children. In this 15-year retrospective clinical study, the SVRI and VIS were all independent predictors of 28-day mortality in pediatric RSS. A lower SVRI and a higher VIS were noted at similar time points in the non-survivors. Furthermore, we identified that SVRI has the most predictive power compared to CI and VIS during the early resuscitative stage of refractory septic shock.

During the early stage of septic shock, clinical presentations may include warm skin, tachycardia, and widened pulse pressure, and warm shock may indicate the hemodynamics of increased CI and decreased SVRI [13]. However, as sepsis progresses, cold shock may develop with decreased CI and increased SVRI. Our serial data showed a clinical course of higher CI along with lower SVRI, and a gradual decrease or normalization in $\mathrm{CI}$ accompanied with an increasing SVRI. Interestingly, under vasoactive-inotropic agent support, the average serial $\mathrm{CI}$ in the mortality group in our cohort reached a normal range of 3.3-6.0 L/min $/ \mathrm{m}^{2}$, which is different to the American College of Critical Care Medicine guidelines, which state that a normal CI indicates a good prognosis [14].

In patients with sepsis, a low SVRI may indicate decreased vasomotor tone caused by endothelial injury, followed by decompensation, leading to an increase in CI levels [18]. Injured endothelial cells indicate dysfunction of the arginine-vasopressin system, which may increase the secretion of tumor necrosis factor, lipopolysaccharides, interleukin-1, circulating endothelin, and nitric oxide, and decrease systemic vascular resistance, causing vascular hyporesponsiveness to vasoactive agents, thereby resulting in refractory hypotension [19]. Previous reports have shown that lower SVRI is associated with mortality in adults with septic shock [20,21]; however, the clinical utilization of SVRI has not been well 
surveyed in pediatric septic shock. Our previous study with the same group reported that SVRI was a predictor for in-hospital mortality in pediatric septic shock [22]. The current study was an expansion of the previous one, which demonstrated the importance of SVRI in the different time points of pediatric septic shock. Our study demonstrated that serial SVRI was significantly lower in the non-survivors. Therefore, early higher levels of SVRI may indicate better outcomes. In the mortality group, although the SVRI increased gradually, the prolonged period of a low SVRI indicated a longer duration of tissue hypoperfusion, representing more organ damage [20]. Moreover, lower serial SVRI may allow the early recognition of disease severity and insufficient treatment and prompt the immediate titration of vasoactive-inotropic agents to raise the SVRI.

Administration of vasoactive-inotropic agents to resuscitate fluid-unresponsive septic shock remains the standard treatment [15]. The VIS may allow for the evaluation of the degree of cardiovascular support, and a high VIS may indicate serious myocardial dysfunction and vasoplegia and serve as a predictor of poor clinical outcomes in children after cardiothoracic surgery and RSS [3,23]. Most patients in both groups used epinephrine or dopamine as the first vasoactive-inotropic agent; however, the VIS quickly increased in the mortality group, and serial VIS was higher. In addition, the clinical benefits of the VIS may include the ease of calculation without the need to analyze prior medical records.

During the first $36 \mathrm{~h}$, the average AUC of SVRI was higher than that of VIS; however, during the period from 42 to $54 \mathrm{~h}$, the result was reversed. The different cutoff values of the SVRI or VIS to predict mortality every $6 \mathrm{~h}$ might confuse physicians when and how to use them probably because of the clinical course of vasoplegia. A significant correlation was observed between the SVRI and VIS, which indicates that vasoplegia could be a predictive factor of septic shock. Vasoplegia was defined as vascular hyporesponsiveness to vasopressors [24]. The symptoms of vasoplegia were commonly observed without thorough hemodynamic data, and the parameters available to clinicians were not sufficient to identify the severity and implications of the condition. [25]. In the present study, we graded the severity of vasoplegia based on the SVRI and VIS. Vasoplegia is a severe condition characterized by persistent vasodilatation despite the use of vasoactive-inotropic agents at high doses. Therefore, the hemodynamics of severe vasoplegia may have low SVRI but high VIS; conversely, mild vasoplegia may have high SVRI but low VIS. Our data demonstrated that low SVRI may initially predict mortality (during the first $36 \mathrm{~h}$ ). Although the SVRI increased gradually (after $36 \mathrm{~h}$ ), children with increasing SVRI under high doses of vasopressors (higher VIS) had mortality (from 42 to $54 \mathrm{~h}$ ). Given the opposite relationship between SVRI and VIS, the intensivists may interpret that the two hemodynamic parameters can predict the progression of vasoplegia in septic shock.

In clinical terms, the cutoff SVRI is divided into three zones: the first zone is for predicting the highest likelihood of mortality (specificity, 100\%), the second zone is for predicting survival (sensitivity, 100\%), and the third zone is indeterminate. The SVRI in predicting mortality at $0 \mathrm{~h}$ was 533 (specificity, 100\%), and the SVRI in predicting survival at $0 \mathrm{~h}$ was 1531 (sensitivity, 100\%). Most children with SVRI < 500 after critical care may indicate a high probability of mortality, whereas most children with SVRI > 1500 may indicate a high probability of survival. The recommended range of SVRI should be more than 500, and $\alpha$-adrenergic agonists, such as epinephrine or norepinephrine, should be titrated immediately to prevent SVRI $<500$. The SVRI in the survivors tended to increase after intensive care. Gradual decreases in SVRI after treatment in the indeterminate zone should be considered alarm signs that require immediate re-evaluation to identify whether or not other factors are present, such as worsening end-organ hypoperfusion and uncontrolled infectious sources.

This study has several limitations. First, this study was executed at a single center with a retrospective design and relatively small sample size. Therefore, information bias may occur. Second, incomplete data regarding macrohemodynamic parameters, such as systolic and diastolic pressures, fluid balance, need for hemodialysis, and serum lactate, were lacking. The lack of this information may prevent the external validation of the 
results of this study. Future studies should address the questions and verify these results prospectively. Third, the PiCCO device needs one central venous catheter and one femoral artery catheter. Placing the femoral artery catheter in younger children is difficult because the diameter of arteries is smaller than that in adults, particularly when they are in shock status. Therefore, we only included older children with the mean age of 12 years, and the youngest patient in this study was 5 years old. Future studies using noninvasive continuous $\mathrm{CO}$ monitoring tools, such as electrical cardiometry, are warranted to verify hemodynamics in neonates and children with septic shock. Fourth, although the Surviving Sepsis Campaign 2020 was recently published, the study period ranged from 2003 to 2017, so the definitions (refractory shock, catecholamine-resistant shock and persistent catecholamine-resistant shock) were based on Surviving Sepsis Campaign 2012, which are the same as Surviving Sepsis Campaign 2020.

\section{Conclusion}

The SVRI and VIS were predictors of mortality in children with catecholamine-RSS. In this study, the SVRI was a powerful predictor of mortality in the early resuscitative stage. Lower serial SVRI may allow for the early recognition of disease severity and strategies to titrate vasoactive-inotropic agents to increase the SVRI.

Author Contributions: E.-P.L., S.-H.H., J.-J.L., S.-L.J., O.-W.C. and H.-P.W. take responsibility for the integrity of the data, interpretation, and analysis. All authors contributed substantially to the study design, data interpretation, and writing of the manuscript. E.-P.L. and S.-H.H. performed the statistical analysis and data synthesis. All authors have read and agreed to the published version of the manuscript.

Funding: The study was supported in part by the China Medical University Hospital (Grant number: C1090903010).

Institutional Review Board Statement: The establishment of the database was approved by the Institutional Review Board of Chang Gung Memorial Hospital (protocol code: 201700079B0D001; date of approval: 5 July 2018).

Informed Consent Statement: Not applicable.

Data Availability Statement: The datasets used and analyzed during the current study are available from the corresponding author on reasonable request.

Acknowledgments: The authors would like to thank the statistician at Chang Gung Memo rial Hospital for completing the statistical analysis.

Conflicts of Interest: The authors declare no conflict of interest.

\section{References}

1. Bone, R.C. The sepsis syndrome definition and general approach to management. Clin. Chest Med. 1996, 17, 175-181. [CrossRef]

2. Goldstein, B.; Giroir, B.; Randolph, A. International consensus conference on pediatric sepsis. international pediatric sepsis consensus conference: Definitions for sepsis and organ dysfunction in pediatrics. Pediatr. Crit. Care Med. 2005, 6, 2-8. [CrossRef]

3. Morin, L.; Ray, S.; Wilson, C.; Remy, S.; Benissa, M.R.; Jansen, N.J.; Javouhey, E.; Peters, M.J.; Kneyber, M.; De Luca, D.; et al. Refractory septic shock in children: A European society of paediatric and neonatal intensive care definition. Intensive Care Med. 2016, 42, 1948-1957. [CrossRef]

4. World Health Organization. Global Health Observatory. Causes of Child Mortality. 2013. Available online: http://www.who.int/ gho/child_health/mortality/causes/en/ (accessed on 31 January 2015).

5. Jawad, I.; Luksic, I.; Rafnsson, S.B. Assessing available information on the burden of sepsis: Global estimates of incidence, prevalence and mortality. J. Glob. Health 2012, 2, 010404. [CrossRef] [PubMed]

6. Weiss, S.L.; Peters, M.J.; Alhazzani, W.; Agus, M.S.D.; Flori, H.R.; Inwald, D.P.; Nadel, S.; Schlapbach, L.J.; Tasker, R.C.; Argent, A.C.; et al. Surviving sepsis campaign international guidelines for the management of septic the management of septic shock and sepsis-associated organ dysfunction in children. Intensive Care Med. 2020, 46 (Suppl. 1), 10-67. [CrossRef] [PubMed]

7. Pollack, M.M.; Fields, A.I.; Ruttimann, U.E. Distributions of cardiopulmonary variables in pediatric survivors and nonsurvivors of septic shock. Crit. Care Med. 1985, 13, 454-459. [CrossRef] [PubMed]

8. Ceneviva, G.; Paschall, J.A.; Maffei, F.; Carcillo, J.A. Hemodynamic support in fluid-refractory pediatric septic shock. Pediatrics 1998, 102, 19. [CrossRef] [PubMed] 
9. Mercier, J.C.; Beaufils, F.; Hartmann, J.F.; Azema, D. Hemodynamic patterns of meningococcal shock in children. Crit. Care Med. 1988, 16, 27-33. [CrossRef]

10. McIntosh, A.; Schmidt, S.; Tong, S.; Deakyne, S.; Davidson, J.; Scott, H. Validation of the vasoactive inotropic score in pediatric sepsis. In Proceedings of the 45th Critical Care Congress of the Society of Critical Care Medicine, Orlando, FL, USA, 20-24 February 2016.

11. Hotchkiss, R.S.; Monneret, G.; Payen, D. Sepsis-induced immunosuppression: From cellular dysfunctions to immunotherapy. Nat. Rev. Immunol. 2013, 13, 862-874. [CrossRef]

12. MacLaren, G.; Butt, W.; Best, D.; Donath, S. Central extracorporeal membrane oxygenation for refractory pediatric septic shock Pediatr. Crit. Care Med. 2011, 12, 133-136. [CrossRef]

13. Biban, P.; Gaffuri, M.; Spaggiari, S.; Zaglia, F.; Serra, A.; Santuz, P. Early recognition and management of septic shock in children. Pediatr. Rep. 2012, 4, 13. [CrossRef] [PubMed]

14. Brierley, J.; Carcillo, J.A.; Choong, K.; Cornell, T.; DeCaen, A.; Deymann, A.; Doctor, A.; Davis, A.; Duff, J.; Dugas, M.A.; et al Clinical practice parameters for hemodynamic support of pediatric and neonatal septic shock: 2007 update from the American college of critical care medicine. Crit. Care Med. 2009, 37, 666-688. [CrossRef] [PubMed]

15. Dellinger, R.P.; Levy, M.M.; Rhodes, A.; Annane, D.; Gerlach, H.; Opal, S.M.; Sevransky, J.E.; Sprung, C.L.; Douglas, I.S.; Jaeschke, R.; et al. International guidelines for management of severe sepsis and septic shock: 2012. Intensive Care Med. 2013, 39, 165-228. [CrossRef] [PubMed]

16. Sankar, J.; Sankar, M.J.; Suresh, C.P.; Dubey, N.K.; Singh, A. Early goal-directed therapy in pediatric septic shock: Comparison of outcomes "with" and "without" intermittent superior venacaval oxygen saturation monitoring: A prospective cohort study. Pediatr Crit. Care Med. 2014, 15, e157-e167. [CrossRef]

17. Gaies, M.G.; Gurney, J.G.; Yen, A.H.; Napoli, M.L.; Gajarski, R.J.; Ohye, R.G.; Charpie, J.R.; Hirsch, J.C. Vasoactive-inotropic score as a predictor of morbidity and mortality in infants after cardiopulmonary bypass. Pediatr. Crit. Care Med. 2010, 11, 234.e8. [CrossRef]

18. Boisramé-Helms, J.; Kremer, H.; Schini-Kerth, V.; Meziani, F. Endothelial dysfunction in sepsis. Curr. Vasc. Pharmacol. 2013, 11, 150-160.

19. Sharshar, T.; Gray, F.; de la Grandmaison, G.L.; Hopklnson, N.S.; Ross, E.; Dorandeu, A.; Orlikowski, D.; Raphael, J.C.; Gajdos, P.; Annane, D. Apoptosis of neurons in cardiovascular autonomic centres triggered by inducible nitric oxide synthase after death from septic shock. Lancet 2003, 362, 1799-1805. [CrossRef]

20. Melo, J.; Peters, J.I. Low systemic vascular resistance: Differential diagnosis and outcome. Crit. Care 1999, 3, 71-77. [CrossRef]

21. Duffy, M.J.; Mullan, B.A.; Craig, T.R.; Shyamsundar, M.; MacSweeney, R.E.; Thompson, G.; Stevenson, M.; McAuley, D.F. Impaired endothelium-depend vasodilatation is a novel predictor of mortality in intensive care. Crit. Care Med. 2011, 39, 629-635. [CrossRef]

22. Lee, E.P.; Chu, S.C.; Hsia, S.H.; Chen, K.F.; Chan, O.W.; Lin, C.Y.; Su, Y.T.; Lin, J.J.; Wu, H.P. Comparison of Predictive Powers for Mortality between Systemic Vascular Resistance Index and Serum Lactate in Children with Persistent Catecholamine-Resistant Shock. BioMed Res. Int. 2020, 2020, 1341326. [CrossRef]

23. Davidson, J.; Tong, S.; Hancock, H.; Hauck, A.; da Cruz, E.; Kaufman, J. Prospective validation of the vasoactive-inotropic score and correlation to short-term outcomes in neonates and infants after cardiothoracic surgery. Intensive Care Med. 2012, 38, 1184-1190. [CrossRef] [PubMed]

24. Lambden, S.; Creagh-Brown, B.C.; Hunt, J.; Summers, C.; Forni, L.G. Definitions and pathophysiology of vasoplegic shock. Crit. Care 2018, 6, 174. [CrossRef] [PubMed]

25. Cecconi, M.; De Backer, D.; Antonelli, M.; Beale, R.; Bakker, J.; Hofer, C.; Jaeschke, R.; Mebazaa, A.; Pinsky, M.R.; Teboul, J.L.; et al Consensus on circulatory shock and hemodynamic monitoring. Task force of the European Society of Intensive Care Medicine. Intensive Care Med. 2014, 40, 1795-1815. [CrossRef] [PubMed] 